\title{
CONSTRUCTION OF SIGN-CHANGING SOLUTIONS FOR A SUBCRITICAL PROBLEM ON THE FOUR DIMENSIONAL HALF SPHERE
}

\author{
RABEH GHOUdi AND KAMAL OULD BOUH
}

(Received September 22, 2014, revised March 5, 2015)

\begin{abstract}
This paper is devoted to studying the nonlinear problem with subcritical exponent $\left(S_{\varepsilon}\right):-\Delta_{g} u+2 u=K|u|^{2-\varepsilon} u$, in $S_{+}^{4}, \partial u / \partial v=0$, on $\partial S_{+}^{4}$, where $g$ is the standard metric of $S_{+}^{4}$ and $K$ is a $C^{3}$ positive Morse function on $\overline{S_{+}^{4}}$. We construct some signchanging solutions which blow up at two different critical points of $K$ in interior. Furthermore, we construct sign-changing solutions of $\left(S_{\mathcal{E}}\right)$ having two bubbles and blowing up at the same critical point of $K$.
\end{abstract}

1. Introduction. We consider the problem of prescribing the scalar curvature under minimal boundary conditions on the standard four dimensional half sphere. More precisely, let $K$ be a $C^{3}$ positive Morse function on $\overline{S_{+}^{4}}$, we look for conditions on $K$ to ensure the existence of solution for the problem

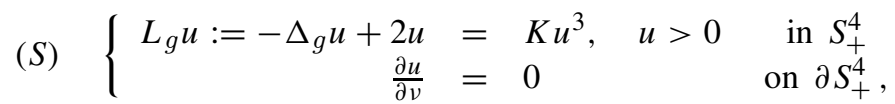

where $g$ is the standard metric of $S_{+}^{n}=\left\{x \in \mathbb{R}^{n+1} /|x|=1, x_{n+1}>0\right\}$.

It is well known that there are topological obstructions of Kazdan-Warner type to solve $(S)$ (see [5]) and so a natural question arises: under which conditions on $K,(S)$ has a solution.

Regarding problem $(S)$, Ben Ayed et al [3] proved that we have a balance phenomenon, that is, the self interaction of the functions failing the Palais-Smale and the interaction of two of those functions are of the same size, if we assume that $(\partial K / \partial \nu)(y)<0$ at any critical point $y$ of $K_{1}=K_{\mid \partial S_{+}^{4}}$. Moreover, it is proved that this phenomenon appears also when the manifold is the three dimensional half sphere (see [6]).

Note that the embedding of $H^{1}\left(S_{+}^{4}\right)$ into $L^{4}\left(S_{+}^{4}\right)$ is noncompact. Hence, for the study of problem $(S)$, it is interesting to approach it by a family of subcritical problems $\left(S_{\varepsilon}\right)$

$$
\left(S_{\varepsilon}\right) \quad\left\{\begin{aligned}
-\Delta_{g} u+2 u & =K|u|^{2-\varepsilon} u, & & \text { in } S_{+}^{4} \\
\frac{\partial u}{\partial v} & =0 & & \text { on } \partial S_{+}^{4},
\end{aligned}\right.
$$

and we need to study the asymptotic behavior of the solutions $\left(u_{\varepsilon}\right)$ as $\varepsilon \rightarrow 0$. Observe that, since $\varepsilon>0$, problem $\left(S_{\varepsilon}\right)$ has always a positive solution $\left(u_{\varepsilon}\right)$. It is well known that, for the minimizing solutions, $\left(u_{\varepsilon}\right)$ has to converge to a solution of $(S)$ or to blow up at one point where the maximum of $K$ or of $K_{1}$ attains.

2010 Mathematics Subject Classification. Primary 35J20; Secondary 35J60.

Key words and phrases. Critical points, Variational problem, Scalar curvature, Bubble-tower solutions. 
For the other solutions $\left(u_{\varepsilon}\right)$ (not minimizing solutions) and in the case of the three dimensional half sphere, Djadli et al [6] proved that $\left(u_{\varepsilon}\right)$ can blow up at $\left\{x_{1}, \ldots, x_{p}\right\}$ such that the points $x_{i}$ 's are different critical points of $K_{1}$ with $(\partial K / \partial v)\left(x_{i}\right)>0$. Furthermore they proved that the $x_{i}$ 's are isolated simple blow ups (see [8] for the definition), which implies that, writing $u_{\varepsilon}=\sum_{i \leq p} \alpha_{i} \delta_{\left(a_{i}, \lambda_{i}\right)}+v_{\varepsilon}$, we have that $\left|a_{i}-a_{j}\right| \geq c>0$ for $i \neq j$ (the function $\delta_{(a, \lambda)}$ is defined in (1.1)). Hence, the tower bubble solutions do not exist. Moreover, In [4] (see also [3]), we proved that there are critical points at infinity (following the terminology of A. Bahri) for the functional associated to the problem $(S)$. This implies the existence of solutions $\left(u_{\varepsilon}\right)$ which blow up at $\left\{y_{1}, \ldots, y_{p}\right\}$ such that the points $y_{i}$ 's are different critical points of $K$ in $S_{+}^{4}$.

In this paper, we aim to construct some sign-changing solutions $\left(u_{\varepsilon}\right)$ of $\left(S_{\varepsilon}\right)$ which blow up at one or two different points in the interior.

Before stating the result, we need to introduce some notations. For $a \in \overline{S_{+}^{4}}$ and $\lambda>0$, let

$$
\delta_{(a, \lambda)}(x)=c_{0} \frac{\lambda}{\left(\lambda^{2}+1+\left(1-\lambda^{2}\right) \cos d(a, x)\right)}
$$

where $d$ is the geodesic distance on $\left(\overline{S_{+}^{4}}, g\right)$ and $c_{0}$ is chosen so that $\delta_{(a, \lambda)}$ is the family of solutions of the following problem

$$
-\Delta u+2 u=u^{3}, \quad u>0, \quad \text { in } S^{4} .
$$

We denote by $P \delta_{(a, \lambda)}$ the projection of the function $\delta_{(a, \lambda)}$ defined by

$$
-\Delta P \delta_{(a, \lambda)}+2 P \delta_{(a, \lambda)}=-\Delta \delta_{(a, \lambda)}+2 \delta_{(a, \lambda)}, \quad \text { in } S_{+}^{4}, \quad \frac{\partial P \delta_{(a, \lambda)}}{\partial v}=0 \text { on } \partial S_{+}^{4} .
$$

It is easy to obtain that $P \delta_{(a, \lambda)}=\delta_{(a, \lambda)}$ if $a \in \partial S_{+}^{4}$.

Let $G$ be the Greens function of $L_{g}:=-\Delta+2 I d$ on $S_{+}^{4}$ and $H$ its regular part defined by

$$
\left\{\begin{array}{c}
G(x, y)=(1-\cos (d(x, y)))^{-1}+H(x, y) \\
L_{g} H=0 \text { in } S_{+}^{4} ; \quad \partial G / \partial v=0 \text { on } \partial S_{+}^{4} .
\end{array}\right.
$$

It is well known that $H$ is a positive function and $H(x, x) \rightarrow+\infty$ as $x$ goes to the boundary.

The space $H^{1}\left(S_{+}^{4}\right)$ is equipped with the norm $\|\cdot\|$ and its corresponding inner product $\langle.,$.$\rangle defined by$

$$
\|u\|^{2}=\int_{S_{+}^{4}}|\nabla u|^{2}+2 \int_{S_{+}^{4}} u^{2}, \quad \text { and } \quad\langle u, v\rangle=\int_{S_{+}^{4}} \nabla u \nabla v+2 \int_{S_{+}^{4}} u v, \quad u, v \in H^{1}\left(S_{+}^{4}\right) .
$$

Our first result deals with the construction of some sign-changing solutions $\left(u_{\varepsilon}\right)$ of $\left(S_{\varepsilon}\right)$ which blow up at two different points in the interior of $S_{+}^{4}$.

THEOREM 1.1. Let $y_{1}$ and $y_{2}$ be nondegenerate critical points of $K$ with $\left(-\Delta K\left(y_{i}\right) /\right.$ $\left.3 K\left(y_{i}\right)-4 H\left(y_{i}, y_{i}\right)\right)>0$ for $i=1,2$. Then, there exists $\varepsilon_{0}>0$ such that for each $\varepsilon \in\left(0, \varepsilon_{0}\right)$, the problem $\left(S_{\varepsilon}\right)$ has a solution $\left(u_{\varepsilon}\right)$ of the form

$$
u_{\varepsilon}=\alpha_{1} P \delta_{\left(x_{1}, \lambda_{1}\right)}-\alpha_{2} P \delta_{\left(x_{2}, \lambda_{2}\right)}+v,
$$


where $\alpha_{i} \rightarrow K\left(y_{i}\right)^{-1 / 2} ;\|v\| \rightarrow 0 ; x_{i} \rightarrow y_{i}, \lambda_{i} \rightarrow+\infty ; \lambda_{1}=\gamma_{1} \lambda_{2}(1+o(1))$ as $\varepsilon \rightarrow 0$. Here, $\gamma_{1}$ is a positive fixed constant.

In the case of positive solutions, the blow up occur with comparable speeds. But for sign-changing solutions, Pistoia and Weth [10] constructed some solutions $\left(u_{\varepsilon}\right)$ of analogous problem of $\left(S_{\varepsilon}\right)$ with many bubbles $\left(u_{\varepsilon}=\sum_{i=1}^{q}(-1)^{i} P \delta_{a_{i}, \lambda_{i}}\right.$, for $\left.q \geq 2\right)$ blowing up at the same point (bubble-tower solutions). This is a new phenomenon for sign-changing solutions compared with the positive one (see [7] and [9]). In our case, we prove that this phenomenon also appear for each $q \geq 2$. In fact, we prove that:

THEOREM 1.2. Assume that $\bar{y}$ is a nondegenerate critical point of $K$ satisfying $(-\Delta K(\bar{y}) / 3 K(\bar{y})-4 H(\bar{y}, \bar{y}))>0$. Then, there exists $\varepsilon_{0}>0$ such that for each $\varepsilon \in\left(0, \varepsilon_{0}\right)$, the problem $\left(S_{\varepsilon}\right)$ has a solution $\left(u_{\varepsilon}\right)$ of the form (1.2) where, as $\varepsilon \rightarrow 0$,

$$
\begin{gathered}
\alpha_{i} \rightarrow K(\bar{y})^{-1 / 2} ;\|v\| \rightarrow 0 ; x_{i} \rightarrow \bar{y}, \lambda_{i} \rightarrow+\infty ; \text { for } i \in\{1,2\} \\
\text { and } \lambda_{1}=\gamma_{2} \lambda_{2}^{3}(1+o(1)) \text { with } \quad \gamma_{2}=2\left(-H(\bar{y}, \bar{y})-\frac{\Delta K(\bar{y})}{12 K(\bar{y})}\right)^{-1} .
\end{gathered}
$$

The remaind of this paper is organized as follows. In Section 2, we recall some preliminaries. In Section 3, we give some careful expansions of gradient of the associated variational functional $I_{\varepsilon}$ for $(\varepsilon>0)$. While Sections 4 and 5 are devoted to the proof of Theorem 1.1 and Theorem 1.2 respectively.

2. Preliminary results. First, let us introduce the general setting. For $\varepsilon>0$, we define the functional

$$
I_{\varepsilon}(u)=\frac{1}{2} \int_{S_{+}^{4}}|\nabla u|^{2}+\int_{S_{+}^{4}} u^{2}-\frac{1}{4-\varepsilon} \int_{S_{+}^{4}} K|u|^{4-\varepsilon}, \quad u \in H^{1}\left(S_{+}^{4}\right) .
$$

Note that if $u$ is a positive critical point of $I_{\varepsilon}$, then $u$ is a solution of $\left(S_{\varepsilon}\right)$, and inversely.

Furthermore, we mention that it will be convenient to perform some stereographic projection in order to reduce our problem to $\mathbb{R}_{+}^{4}$. Let $D^{1,2}\left(\mathbb{R}_{+}^{4}\right)$ denote the completion of $C_{c}^{\infty}\left(\overline{\mathbb{R}_{+}^{4}}\right)$ with respect to Dirichlet norm. The stereographic projection $\pi_{a}$ through a point $a \in \partial S_{+}^{4}$ induces an isometry $1: H^{1}\left(S_{+}^{4}\right) \rightarrow D^{1,2}\left(\mathbb{R}_{+}^{4}\right)$ according to the following formula

$$
(1 v)(x)=\left(\frac{2}{1+|x|^{2}}\right) v\left(\pi_{a}^{-1}(x)\right), \quad v \in H^{1}\left(S_{+}^{4}\right), x \in \mathbb{R}_{+}^{4} .
$$

In particular, one can check that the following holds true, for every $v \in H^{1}\left(S_{+}^{4}\right)$

$$
\int_{S_{+}^{4}}\left(|\nabla v|^{2}+2 v^{2}\right)=\int_{\mathbb{R}_{+}^{4}}|\nabla(1 v)|^{2} \quad \text { and } \int_{S_{+}^{4}}|v|^{4}=\int_{\mathbb{R}_{+}^{4}}|1 v|^{4} .
$$

In the sequel, we will identify the function $K$ and its composition with the stereographic projection $\pi_{a}$. We will also identify a point $b$ of $S_{+}^{4}$ and its image by $\pi_{a}$. Moreover, it is easy to see that, by (2.2) with $\pi_{-a}$, the function $1 \delta_{(a, \lambda)}$ is equal to

$$
1 \delta_{(a, \lambda)}=c_{0} \frac{\lambda}{1+\lambda^{2}|x-a|^{2}} .
$$


For sake of simplicity, we will write $\delta_{(a, \lambda)}$ instead of $1 \delta_{(a, \lambda)}$. These facts will be assumed in the sequel.

LEMma 2.1 ([3]). For $a \in \partial S_{+}^{4}$, we have $\left(\partial \delta_{(a, \lambda)}\right) /(\partial v)=0$ and $\delta_{(a, \lambda)}=P \delta_{(a, \lambda)}$. For $a \in S_{+}^{4}$, we have

such that $f_{(a, \lambda)}$ satisfies

$$
P \delta_{(a, \lambda)}-\delta_{(a, \lambda)}=c_{0} \frac{H(a, .)}{\lambda}+f_{(a, \lambda)}
$$

$$
\left|f_{(a, \lambda)}\right|_{L^{\infty}} \leq \frac{c}{\lambda^{3} d^{4}} \quad \text { and } \quad \lambda \frac{\partial f}{\partial \lambda}=O\left(\frac{1}{\lambda^{3} d^{4}}\right),
$$

where $d=d\left(a, \partial S_{+}^{4}\right)$.

PROOF. We will repeat the proof here to be self-contained.

Using a stereographic projection, we are led to prove the corresponding estimates on $\mathbb{R}_{+}^{4}$. We still denote by $G$ and $H$ the Greens function and its regular part of the Laplacian on $\mathbb{R}_{+}^{4}$ under Neumann boundary conditions. In this case, we have

$$
\delta_{(a, \lambda)}=c_{0} \frac{\lambda}{1+\lambda^{2}|x-a|^{2}} \text { and } H(a, x)=\frac{1}{|\bar{a}-x|^{2}},
$$

where $\bar{a}$ is the symmetric of a with respect to $\partial \mathbb{R}_{+}^{4}$.

Observe that, for $f_{(a, \lambda)}=P \delta_{(a, \lambda)}-\delta_{(a, \lambda)}-H(a,.) / \lambda$,

$$
\Delta f_{(a, \lambda)}=0 \text { in } \mathbb{R}_{+}^{4}, \quad \frac{\partial f_{(a, \lambda)}}{\partial v}=\frac{\partial \delta}{\partial v}-\frac{1}{\lambda} \frac{\partial H}{\partial v}=O\left(\frac{1}{\lambda^{3} d^{5}}\right) .
$$

Thus, using the Green formula, we derive

$$
f_{(a, \lambda)}(x)=c \int_{\partial \mathbb{R}_{+}^{4}} G\left(\frac{\partial \delta}{\partial v}-\frac{1}{\lambda} \frac{\partial H}{\partial v}\right) \leq \frac{c^{\prime}}{\lambda^{3} d_{a}^{2}} \int_{\partial \mathbb{R}_{+}^{4}} G \frac{1}{|a-x|^{3}},
$$

where $d_{a}$ is the distance of $a$ to the boundary. $G$ satisfies

$$
\int_{\partial \mathbb{R}_{+}^{4}} G(x, y) \frac{1}{|a-x|^{3}}=O\left(\frac{1}{d_{a}^{2}}\right) .
$$

Let

$$
E_{(x, \lambda)}=\left\{w \in H^{1}\left(S_{+}^{4}\right) /\langle w, \varphi\rangle=0 \quad \forall \varphi \in \operatorname{Span}\left\{P \delta_{i}, \frac{\partial P \delta_{i}}{\partial \lambda_{i}}, \frac{\partial P \delta_{i}}{\partial x_{i}^{j}}, i=1,2 ; j \leq 4\right\}\right\} .
$$

Here, $x_{i}^{j}$ denotes the $j$-th component of $x_{i}$. For sake of simplicity, we will write $P \delta_{i}$ instead of $P \delta_{\left(x_{i}, \lambda_{i}\right)}$ and therefore, for $u=\alpha_{1} P \delta_{\left(x_{1}, \lambda_{1}\right)}-\alpha_{2} P \delta_{\left(x_{2}, \lambda_{2}\right)}+v$ we can write $u=\alpha_{1} P \delta_{1}-$ $\alpha_{2} P \delta_{2}+v$.

3. Expansions of the gradient of the functional $I_{\varepsilon}$. In this section, we collect some expansions of the gradient of the functional $I_{\varepsilon}$ associated to the problem $\left(S_{\varepsilon}\right)$ for $\varepsilon>0$ which are needed in Section 4 . We start by giving the following remark which is proved in [11] when $S_{+}^{4}$ is replaced by a bounded domain of $\mathbb{R}^{3}$. 
REMARK 3.1. Let $\delta_{(a, \lambda)}$ be the function defined in (1.1). Assume that $\varepsilon \log \lambda$ is small enough. For $\varepsilon>0$, we have

$$
\delta_{(a, \lambda)}^{-\varepsilon}(x)=1-\varepsilon \log \delta_{(a, \lambda)}+O\left(\varepsilon^{2} \log ^{2} \lambda\right) \quad \text { in } S_{+}^{4} .
$$

Now, explicit computations, by Remark 3.1 and Lemma 2.1, yield the following propositions

Proposition 3.2. For $u=\alpha_{1} P \delta_{\left(x_{1}, \lambda_{1}\right)}-\alpha_{2} P \delta_{\left(x_{2}, \lambda_{2}\right)}+v$, with $v \in E_{x, \lambda}$, we have

$$
\left\langle\nabla I_{\varepsilon}, P \delta_{i}\right\rangle=(-1)^{i+1} \alpha_{i} S_{4}\left(1-\alpha_{i}^{2-\varepsilon} K\left(x_{i}\right)\right)+O\left(\varepsilon \log \lambda_{i}+\frac{1}{\lambda_{2}^{2}}+\varepsilon_{12}+\|v\|^{2}\right),
$$

where

$$
S_{4}=64 \int_{\mathbb{R}^{4}} \frac{d x}{\left(1+|x|^{2}\right)^{4}}
$$

Proof. We have

$$
\left\langle\nabla I_{\varepsilon}, h\right\rangle=\int_{S_{+}^{4}} \nabla u \nabla h+2 \int_{S_{+}^{4}} u h-\int_{S_{+}^{4}} K|u|^{2-\varepsilon} u h .
$$

A computation similar to the one performed in [1] shows that

$$
\left\|P \delta_{i}\right\|^{2}=\int_{\mathbb{R}_{+}^{4}}\left|\nabla P \delta_{i}\right|^{2}=S_{4}+O\left(\frac{1}{\lambda_{i}^{2}}\right),
$$

and

$$
\int_{S_{+}^{4}} \nabla P \delta_{1} \nabla P \delta_{2}+2 \int_{S_{+}^{4}} P \delta_{1} P \delta_{2}=\int_{\mathbb{R}_{+}^{4}} \nabla P \delta_{1} \nabla P \delta_{2}=\int_{\mathbb{R}_{+}^{4}} \delta_{1}^{3} P \delta_{2}=O\left(\varepsilon_{12}\right) .
$$

For the integral, we write

$\int_{S_{+}^{4}} K|u|^{2-\varepsilon} u P \delta_{1}=\int_{S_{+}^{4}} K\left|\alpha_{1} P \delta_{1}-\alpha_{2} P \delta_{2}\right|^{2-\varepsilon}\left(\alpha_{1} P \delta_{1}-\alpha_{2} P \delta_{2}\right) P \delta_{1}+O\left(\varepsilon_{12}^{2} \log \varepsilon_{12}^{-1}+|v|^{2}\right)$.

We also write

$$
\begin{gathered}
\int_{S_{+}^{4}} K\left|\alpha_{1} P \delta_{1}-\alpha_{2} P \delta_{2}\right|^{2-\varepsilon}\left(\alpha_{1} P \delta_{1}-\alpha_{2} P \delta_{2}\right) P \delta_{1}=\alpha_{1}^{3-\varepsilon} \int_{S_{+}^{4}} K P \delta_{1}^{4-\varepsilon}-\alpha_{2}^{3-\varepsilon} \int_{S_{+}^{4}} K P \delta_{2}^{3-\varepsilon} P \delta_{1} \\
-(3-\varepsilon) \alpha_{1}^{2-\varepsilon} \alpha_{2} \int_{S_{+}^{4}} K P \delta_{1}^{3-\varepsilon} P \delta_{2}+O\left(\varepsilon_{12}^{2} \log \varepsilon_{12}^{-1}\right) .
\end{gathered}
$$

Expanding of $K$ around $x_{1}$ and $x_{2}$, we get

$$
\begin{aligned}
& \int_{S_{+}^{4}} K P \delta_{i}^{4-\varepsilon}=\int_{\mathbb{R}_{+}^{4}} K P \delta_{i}^{4-\varepsilon}=K\left(x_{i}\right) S_{4}+O\left(\varepsilon \log \lambda_{i}+\frac{1}{\lambda_{i}^{2}}\right), \\
& \int_{S_{+}^{4}} K P \delta_{2}^{3-\varepsilon} P \delta_{1}=\int_{\mathbb{R}_{+}^{4}} K P \delta_{2}^{3-\varepsilon} P \delta_{1}=O\left(\varepsilon \log \lambda_{2}+\varepsilon_{12}\right), \\
& \int_{S_{+}^{4}} K P \delta_{1}^{3-\varepsilon} P \delta_{2}=\int_{\mathbb{R}_{+}^{4}} K P \delta_{1}^{3-\varepsilon} P \delta_{2}=O\left(\varepsilon \log \lambda_{1}+\varepsilon_{12}\right) .
\end{aligned}
$$

Combining (3.1)-(3.8), we easily derive our proposition. 
Proposition 3.3. For $u=\alpha_{1} P \delta_{\left(x_{1}, \lambda_{1}\right)}-\alpha_{2} P \delta_{\left(x_{2}, \lambda_{2}\right)}+v$, with $v \in E_{x, \lambda}$, we have the following expansion:

$$
\begin{aligned}
\left\langle\nabla I_{\varepsilon}(u), \lambda_{i} \frac{\partial P \delta_{i}}{\partial \lambda_{i}}\right\rangle & =(-1)^{i+1}\left[\alpha_{j} \frac{c_{2}}{2}\left(\alpha_{i}^{2-\varepsilon} K\left(x_{i}\right)+\alpha_{j}^{2-\varepsilon} K\left(x_{j}\right)-1\right)\left(\lambda_{i} \frac{\partial \varepsilon_{12}}{\partial \lambda_{i}}-2 \frac{H\left(x_{1}, x_{2}\right)}{\lambda_{1} \lambda_{2}}\right)\right. \\
& \left.+\alpha_{i}^{3-\varepsilon} \frac{\varepsilon S_{4} K\left(x_{i}\right)}{4}+\alpha_{i}^{3-\varepsilon} \frac{c_{2}}{12} \frac{\Delta K\left(x_{i}\right)}{\lambda_{i}^{2}}+\alpha_{i} c_{2} \frac{H\left(x_{i}, x_{i}\right)}{\lambda_{i}^{2}}\left(2 \alpha_{i}^{2-\varepsilon} K\left(x_{i}\right)-1\right)\right] \\
& +O\left(\varepsilon^{2} \log \lambda_{i}+\frac{\varepsilon \log \lambda_{i}}{\lambda_{i}^{2}}+\frac{1}{\lambda_{i}^{3}}+\|v\|^{2}\right) \\
& +O\left(\varepsilon \varepsilon_{12}\left(\log \varepsilon_{12}^{-1}\right)^{\frac{1}{2}}+\varepsilon_{12}^{2} \log \varepsilon_{12}^{-1}+\varepsilon_{12}\left(\log \varepsilon_{12}^{-1}\right)^{\frac{1}{2}}\left(\frac{1}{\lambda_{1}}+\frac{1}{\lambda_{2}}\right)\right),
\end{aligned}
$$

where

$$
c_{1}=64 \int_{\mathbb{R}_{+}^{4}} \frac{x_{4}\left(|x|^{2}-1\right)}{\left(1+|x|^{2}\right)^{5}} d x, \quad c_{2}=64 \int_{\mathbb{R}^{4}} \frac{d x}{\left(1+|x|^{2}\right)^{3}} .
$$

ProOF. Observe that (see [1])

$$
\int_{\mathbb{R}_{+}^{4}} \nabla P \delta_{i} \nabla\left(\lambda_{i} \frac{\partial P \delta_{i}}{\partial \lambda_{i}}\right)=-c_{2} \frac{H\left(x_{1}, x_{2}\right)}{\lambda_{i}^{2}}+O\left(\frac{1}{\lambda_{i}^{3}}\right)
$$

$$
\int_{\mathbb{R}_{+}^{4}} \nabla P \delta_{j} \nabla\left(\lambda_{i} \frac{\partial \delta_{i}}{\partial \lambda_{i}}\right)=\int_{\mathbb{R}_{+}^{4}} \delta_{j}^{3} \lambda_{i} \frac{\partial \delta_{i}}{\partial \lambda_{i}}=\frac{c_{2}}{2}\left(\lambda_{i} \frac{\partial \varepsilon_{12}}{\partial \lambda_{i}}-2 \frac{H\left(x_{1}, x_{2}\right)}{\lambda_{1} \lambda_{2}}\right)+O\left(\varepsilon_{12}^{2} \log \left(\varepsilon_{12}^{-1}\right)\right) .
$$

For the other part

$$
\begin{aligned}
\int_{\mathbb{R}_{+}^{4}} K P \delta_{i}^{3-\varepsilon} \lambda_{i} \frac{\partial P \delta_{i}}{\partial \lambda_{i}}= & -\frac{c_{2}}{\lambda_{i}^{2}} \frac{\Delta K\left(x_{i}\right)}{12}-\frac{S_{4} \varepsilon}{4} K\left(x_{i}\right)-2 c_{2} K\left(x_{i}\right) \frac{H\left(x_{i}, x_{i}\right)}{\lambda_{i}^{2}} \\
& +O\left(\varepsilon^{2} \log \lambda_{i}+\frac{1}{\lambda_{i}^{3}}+\frac{\varepsilon \log \lambda_{i}}{\lambda_{i}^{2}}\right), \\
\int_{\mathbb{R}_{+}^{4}} K P \delta_{j}^{3-\varepsilon} \lambda_{i} \frac{\partial P \delta_{i}}{\partial \lambda_{i}}= & K\left(x_{j}\right) \frac{c_{2}}{2}\left(\lambda_{i} \frac{\partial \varepsilon_{12}}{\partial \lambda_{i}}-2 \frac{H\left(x_{1}, x_{2}\right)}{\lambda_{1} \lambda_{2}}\right)+O\left(\varepsilon \varepsilon_{12}\left(\log \left(\varepsilon_{12}^{-1}\right)\right)^{\frac{1}{2}}+\frac{1}{\lambda_{i}^{3}}\right) \\
& +O\left(\varepsilon_{12}^{2} \log \left(\varepsilon_{12}^{-1}\right)\right),
\end{aligned}
$$

$(3-\varepsilon) \int_{\mathbb{R}_{+}^{4}} K P \delta_{i}^{2-\varepsilon} P \delta_{j} \lambda_{i} \frac{\partial P \delta_{i}}{\partial \lambda_{i}}=K\left(x_{i}\right) \frac{c_{2}}{2}\left(\lambda_{i} \frac{\partial \varepsilon_{12}}{\partial \lambda_{i}}-2 \frac{H\left(x_{1}, x_{2}\right)}{\lambda_{1} \lambda_{2}}\right)+O\left(\varepsilon \varepsilon_{12}\left(\log \left(\varepsilon_{12}^{-1}\right)\right)^{\frac{1}{2}}\right)$

$$
+O\left(\varepsilon_{12}^{2} \log \left(\varepsilon_{12}^{-1}\right)+\frac{\varepsilon_{12}}{\lambda_{i}}\left(\log \left(\varepsilon_{12}^{-1}\right)\right)^{\frac{1}{2}}\right) .
$$

Combining (3.1) and (3.9)-(3.13), we derive our proposition.

Proposition 3.4. For $u=\alpha_{1} P \delta_{\left(x_{1}, \lambda_{1}\right)}-\alpha_{2} P \delta_{\left(x_{2}, \lambda_{2}\right)}+v$, with $v \in E_{x, \lambda}$, we have

$$
\left\langle\nabla I_{\varepsilon}(u), \frac{1}{\lambda_{i}} \frac{\partial P \delta_{i}}{\partial x_{i}}\right\rangle=\frac{(-1)^{i+1}}{\lambda_{i}}\left[\alpha_{i} c_{2}\left(1-\sum \alpha_{j}^{2-\varepsilon} K\left(x_{j}\right)\right)\left(\frac{\partial \varepsilon_{12}}{\partial x_{i}}+\frac{2}{\lambda_{1} \lambda_{2}} \frac{\partial H\left(x_{1}, x_{2}\right)}{\partial x_{i}}\right)\right.
$$




$$
\begin{aligned}
& \left.-\alpha_{i}^{3-\varepsilon} c_{3} \nabla K\left(x_{i}\right)+\left(1-\alpha_{i}^{2-\varepsilon} K\left(x_{i}\right)\right) \frac{\alpha_{i} c_{2}}{\lambda_{i}^{2}} \frac{\partial H\left(x_{i}, x_{i}\right)}{\partial x_{i}}\right] \\
& +O\left(\frac{\varepsilon \log \lambda_{i}}{\lambda_{i}}\left|\nabla K\left(x_{i}\right)\right|+\frac{1}{\lambda_{i}^{2}}+\|v\|^{2}+\lambda_{j}\left|x_{1}-x_{2}\right| \varepsilon_{12}^{5 / 2}\right) \\
& +O\left(\varepsilon \varepsilon_{12}\left(\log \varepsilon_{12}^{-1}\right)^{\frac{1}{2}}+\varepsilon_{12}^{2} \log \varepsilon_{12}^{-1}+\varepsilon_{12}\left(\log \varepsilon_{12}^{-1}\right)^{\frac{1}{2}}\left(\frac{1}{\lambda_{1}}+\frac{1}{\lambda_{2}}\right)\right),
\end{aligned}
$$

where

$$
c_{3}=16 \int_{\mathbb{R}^{4}} \frac{x_{4}^{2}}{\left(1+|x|^{2}\right)^{5}} d x, \quad c_{4}=132 \int_{\mathbb{R}_{+}^{4}} \frac{x_{4}}{\left(1+|x|^{2}\right)^{5}} d x .
$$

PROOF. An easy computation shows

$$
\int_{\mathbb{R}_{+}^{4}} \nabla P \delta_{i} \nabla\left(\frac{1}{\lambda_{i}} \frac{\partial P \delta_{i}}{\partial x_{i}}\right)=\frac{c_{2}}{\lambda_{i}^{3}} \frac{\partial H\left(x_{i}, x_{i}\right)}{\partial x_{i}}+\left(\frac{1}{\lambda_{i}^{4}}\right),
$$

$$
\begin{aligned}
\int_{\mathbb{R}_{+}^{4}} \nabla P \delta_{j} & \nabla\left(\frac{1}{\lambda_{i}} \frac{\partial \delta_{i}}{\partial x_{i}}\right)=\int_{\mathbb{R}_{+}^{4}} \delta_{j}^{3} \frac{1}{\lambda_{i}} \frac{\partial P \delta_{i}}{\partial x_{i}} \\
= & \frac{c_{2}}{\lambda_{i}}\left(\frac{\partial \varepsilon_{12}}{\partial x_{i}}+\frac{2}{\lambda_{1} \lambda_{2}} \frac{\partial H\left(x_{1}, x_{2}\right)}{\partial x_{i}}\right)+O\left(\varepsilon_{12}^{2} \log \left(\varepsilon_{12}^{-1}\right)+\varepsilon_{12}^{\frac{5}{2}} \lambda_{i}\left|x_{1}-x_{2}\right|\right) .
\end{aligned}
$$

For the other part

$$
\int_{\mathbb{R}_{+}^{4}} K P \delta_{i}^{3-\varepsilon} \frac{1}{\lambda_{i}} \frac{\partial P \delta_{i}}{\partial x_{i}}=c_{3} \frac{\nabla K\left(x_{i}\right)}{\lambda_{i}}+O\left(\frac{1}{\lambda_{i}^{2}}+\varepsilon^{2} \log ^{2} \lambda_{i}\right),
$$

$$
\begin{aligned}
& \int_{\mathbb{R}_{+}^{4}} K P \delta_{j}^{3-\varepsilon} \frac{1}{\lambda_{i}} \frac{\partial P \delta_{i}}{\partial x_{i}}=K\left(x_{j}\right) \frac{c_{2}}{\lambda_{i}}\left(\frac{\partial \varepsilon_{12}}{\partial x_{i}}+\frac{2}{\lambda_{1} \lambda_{2}} \frac{\partial H\left(x_{1}, x_{2}\right)}{\partial x_{i}}\right)+O\left(\varepsilon_{12}^{\frac{5}{2}} \lambda_{i}\left|x_{1}-x_{2}\right|\right) \\
& +O\left(\varepsilon_{12}^{2} \log \left(\varepsilon_{12}^{-1}\right)+\frac{1}{\lambda_{i}} \varepsilon_{12}\left(\log \left(\varepsilon_{12}^{-1}\right)\right)^{\frac{1}{2}}\right), \\
& (3-\varepsilon) \int_{\mathbb{R}_{+}^{4}} K P \delta_{i}^{2-\varepsilon} P \delta_{j} \frac{1}{\lambda_{i}} \frac{\partial P \delta_{i}}{\partial x_{i}}=K\left(x_{i}\right) \frac{c_{2}}{\lambda_{i}}\left(\frac{\partial \varepsilon_{12}}{\partial x_{i}}+\frac{2}{\lambda_{1} \lambda_{2}} \frac{\partial H\left(x_{1}, x_{2}\right)}{\partial x_{i}}\right)+O\left(\varepsilon_{12}^{\frac{5}{2}} \lambda_{2}\left|x_{1}-x_{2}\right|\right) \\
& +O\left(\varepsilon_{12}^{2} \log \left(\varepsilon_{12}^{-1}\right)+\frac{1}{\lambda_{i}} \varepsilon_{12}\left(\log \left(\varepsilon_{12}^{-1}\right)\right)^{\frac{1}{2}}\right) .
\end{aligned}
$$

Using (3.1), (3.14)-(3.18), we have our proposition.

\section{Proof of Theorem 1.1. Let}

$$
\begin{aligned}
M_{\varepsilon, 1}= & \left\{m=\left(\alpha, \lambda, x_{1}, x_{2}, v\right) \in \mathbb{R}^{2} \times\left(\mathbb{R}_{+}^{*}\right)^{2} \times S_{+}^{4} \times S_{+}^{4} \times H^{1}\left(S_{+}^{4}\right): v \in E_{(x, \lambda)},\|v\|<v_{0} ;\right. \\
& \left.\left|\frac{\alpha_{i}^{2} K\left(x_{i}\right)}{\alpha_{j}^{2} K\left(x_{j}\right)}-1\right|<v_{0}, \lambda_{i}>\frac{1}{\nu_{0}}, \varepsilon \log \lambda_{i}<v_{0}, \forall i ; c_{0}<\frac{\lambda_{1}}{\lambda_{2}}<c_{0}^{-1} ;\left|x_{1}-x_{2}\right|>d_{0}\right\},
\end{aligned}
$$


where $\sigma, c_{0}, d_{0}$ are some suitable positive constants, $\nu_{0}$ is a small positive constant. Let us define the function by

$$
\Psi_{\varepsilon, 1}: M_{\varepsilon, 1} \rightarrow \mathbb{R} ; \quad m=(\alpha, \lambda, x, v) \mapsto I_{\varepsilon}\left(\alpha_{1} P \delta_{\left(x_{1}, \lambda_{1}\right)}-\alpha_{2} P \delta_{\left(x_{2}, \lambda_{2}\right)}+v\right) .
$$

As in [2], using the Euler-Lagrange's coefficients, we easily get the following proposition.

Proposition 4.1. Let $m=(\alpha, \lambda, x, v) \in M_{\varepsilon, 1} . m$ is a critical point of $\Psi_{\varepsilon, 1}$ if and only if $u=\alpha_{1} P \delta_{1}-\alpha_{2} P \delta_{2}+v$ is a critical point of $I_{\varepsilon}$, i.e. if and only if there exists $(A, B, C) \in \mathbb{R}^{2} \times \mathbb{R}^{2} \times\left(\mathbb{R}^{4}\right)^{2}$ such that the following holds:

$$
\begin{aligned}
& \left(E_{\alpha_{i}}\right) \frac{\partial \Psi_{\varepsilon, 1}}{\partial \alpha_{i}}=0, \forall i=1,2 \\
& \left(E_{\lambda_{i}}\right) \frac{\partial \Psi_{\varepsilon, 1}}{\partial \lambda_{i}}=B_{i}\left\langle\frac{\partial^{2} P \delta_{i}}{\partial \lambda_{i}^{2}}, v\right\rangle+\sum_{j=1}^{4} C_{i j}\left\langle\frac{\partial^{2} P \delta_{i}}{\partial x_{i}^{j} \partial \lambda_{i}}, v\right\rangle, \forall i=1,2 \\
& \left(E_{x_{i}}\right) \frac{\partial \Psi_{\varepsilon, 1}}{\partial x_{i}}=B_{i}\left\langle\frac{\partial^{2} P \delta_{i}}{\partial \lambda_{i} \partial x_{i}}, v\right\rangle+\sum_{j=1}^{4} C_{i j}\left\langle\frac{\partial^{2} P \delta_{i}}{\partial x_{i}^{j} \partial x_{i}}, v\right\rangle, \forall i=1,2 \\
& \left(E_{v}\right) \frac{\partial \Psi_{\varepsilon, 1}}{\partial v}=\sum_{i=1,2}\left(A_{i} P \delta_{i}+B_{i} \frac{\partial P \delta_{i}}{\partial \lambda_{i}}+\sum_{j=1}^{4} C_{i j} \frac{\partial P \delta_{i}}{\partial x_{i}^{j}}\right) .
\end{aligned}
$$

The results of Theorem 1.1 will be obtained through a careful analysis of (4.2)-(4.5) on $M_{\varepsilon, 1}$. As usual in this type of problems, we first deal with the $v$-part of $u$, in order to show that it is negligible with respect to the concentration phenomenon. The study of $\left(E_{v}\right)$ yields:

PROPOSITION 4.2. There exists a smooth map which to any $(\varepsilon, \alpha, \lambda, x)$ such that $(\alpha, \lambda, x, 0)$ in $M_{\varepsilon, 1}$ associates $\bar{v} \in E_{(x, \lambda)}$ such that $\|v\|<v_{0}$ and $\left(E_{v}\right)$ is satisfied for some $(A, B, C) \in \mathbb{R}^{2} \times \mathbb{R}^{2} \times\left(\mathbb{R}^{4}\right)^{2}$. Such a $\bar{v}$ is unique, minimizes $\Psi_{\varepsilon, 1}(\alpha, \lambda, x, v)$ with respect to $v$ in $\left\{v \in E_{(x, \lambda)} /\|v\|<v_{0}\right\}$, and we have the following estimate

$$
\|\bar{v}\|=O\left(\varepsilon+\sum\left(\frac{\left|\nabla K\left(x_{i}\right)\right|}{\lambda_{i}}+\frac{1}{\lambda_{i}^{2}}\right)+\varepsilon_{12}\left(\log \varepsilon_{12}^{-1}\right)^{1 / 2}\right) .
$$

PROOF. Expanding $I_{\varepsilon}$ with respect to $v \in E_{(x, \lambda)}$, we obtain

$$
I_{\varepsilon}\left(\alpha_{1} P \delta_{1}-\alpha_{2} P \delta_{2}+v\right)=c(\alpha, x, \lambda)+\frac{1}{2} Q(v, v)-f(v)+R(v),
$$

where $Q(.,$.$) is a quadratic form positive definite, f($.$) is a linear form and R(v)$ satisfies $R(v)=o\left(\|v\|^{2}\right), R^{\prime}(v)=o(\|v\|)$ and $R^{\prime \prime}(v)=o(1)$.

Since $Q(v, v)$ is positive definite, we derive that the following problem

$$
\min \left\{I_{\varepsilon}\left(\alpha_{1} P \delta_{1}-\alpha_{2} P \delta_{2}+v\right), v \in E_{(x, \lambda)} \text { and }\|v\|<v_{0}\right\}
$$

is achieved by a unique function $\bar{v}$ which satisfies $\|\bar{v}\| \leq c\|f\|$. Now, following [3] we get the estimate (4.6). Since $\bar{v}$ is orthogonal to the functions $\left\{P \delta_{i}, \partial P \delta_{i} / \partial \lambda_{i}, \partial P \delta_{i} / \partial x_{i}^{j}, i \leq 2, j \leq\right.$ 4\}, there exist $A, B$ and $C$ such that 


$$
\begin{aligned}
\frac{\partial \Psi_{\varepsilon, 1}}{\partial v}(\alpha, \lambda, x, \bar{v}) & =\nabla I_{\varepsilon}\left(\alpha_{1} P \delta_{1}-\alpha_{2} P \delta_{2}+\bar{v}\right) \\
& =\sum_{i=1,2}\left(A_{i} P \delta_{i}+B_{i} \frac{\partial P \delta_{i}}{\partial \lambda_{i}}+\sum_{j=1}^{4} C_{i j} \frac{\partial P \delta_{i}}{\partial x_{i}^{j}}\right)
\end{aligned}
$$

The proposition follows.

Proof of Theorem 1.1. Once $\bar{v}$ is defined by Proposition 4.2, we estimate the corresponding numbers $A, B, C$ by taking the scalar product in $H^{1}\left(S_{+}^{4}\right)$ of $\left(E_{v}\right)$ with $P \delta_{1}, P \delta_{2}$, $\partial P \delta_{1} / \partial \lambda_{1}, \partial P \delta_{2} / \partial \lambda_{2}, \partial P \delta_{1} / \partial x_{1}$ and $\partial P \delta_{2} / \partial x_{2}$ respectively. Thus we get a quasi-diagonal system whose coefficients are given by

$$
\begin{aligned}
& \int_{\mathbb{R}_{+}^{4}}\left|\nabla P \delta_{i}\right|^{2}=S_{4}+O\left(\frac{1}{\lambda_{i}^{2}}\right) ; \quad \int_{\mathbb{R}_{+}^{4}} \nabla P \delta_{i} \nabla P \delta_{j}=O\left(\frac{1}{\lambda_{i} \lambda_{j}}\right) ; \quad \int_{\mathbb{R}_{+}^{4}} \nabla P \delta_{i} \nabla \frac{\partial P \delta_{i}}{\partial \lambda_{i}}=O\left(\frac{1}{\lambda_{i}^{3}}\right), \\
& \int_{\mathbb{R}_{+}^{4}} \nabla P \delta_{i} \nabla \frac{\partial P \delta_{j}}{\partial \lambda_{j}}=O\left(\frac{1}{\lambda_{i} \lambda_{j}^{2}}\right) ; \int_{\mathbb{R}_{+}^{4}}\left|\nabla \frac{\partial P \delta_{i}}{\partial \lambda_{i}}\right|^{2}=\frac{\Gamma_{1}}{\lambda_{i}^{2}}+O\left(\frac{1}{\lambda_{i}^{3}}\right) ; \int_{\mathbb{R}_{+}^{4}} \nabla \frac{\partial P \delta_{i}}{\partial \lambda_{i}} \nabla \frac{\partial P \delta_{j}}{\partial \lambda_{j}}=O\left(\frac{1}{\lambda_{i}^{2} \lambda_{j}^{2}}\right), \\
& \int_{\mathbb{R}_{+}^{4}} \nabla \frac{\partial P \delta_{i}}{\partial \lambda_{i}} \nabla \frac{\partial P \delta_{i}}{\partial x_{i}}=O\left(\frac{1}{\lambda_{i}^{3}}\right) ; \quad \int_{\mathbb{R}_{+}^{4}}\left|\nabla \frac{\partial P \delta_{i}}{\partial x_{i}}\right|^{2}=\Gamma_{2} \lambda_{i}^{2}+O\left(\frac{1}{\lambda_{i}}\right) ; \int_{\mathbb{R}_{+}^{4}} \nabla P \delta_{i} \nabla \frac{\partial P \delta_{i}}{\partial x_{i}}=O\left(\frac{1}{\lambda_{i}^{2}}\right), \\
& \int_{\mathbb{R}_{+}^{4}} \nabla P \delta_{i} \nabla \frac{\partial P \delta_{j}}{\partial x_{j}}=O\left(\frac{1}{\lambda_{i}}\right) ; \quad \int_{\mathbb{R}_{+}^{4}} \nabla \frac{\partial P \delta_{i}}{\partial x_{i}} \nabla \frac{\partial P \delta_{j}}{\partial x_{j}}=O\left(\frac{1}{\lambda_{i}}\right),
\end{aligned}
$$

with $\Gamma_{1}, \Gamma_{2}$ are positive constants and where we have used the fact that $\left|x_{1}-x_{2}\right| \geq c>0$. The other hand side is given by (4.10)

$$
(-1)^{i} \frac{\partial \Psi_{\varepsilon, 1}}{\partial \alpha_{i}}=\left\langle\frac{\partial \Psi_{\varepsilon, 1}}{\partial v}, P \delta_{i}\right) ; \frac{(-1)^{i}}{\alpha_{i}} \frac{\partial \Psi_{\varepsilon, 1}}{\partial \lambda_{i}}=\left\langle\frac{\partial \Psi_{\varepsilon, 1}}{\partial v}, \frac{\partial P \delta_{i}}{\partial \lambda_{i}}\right\rangle ; \frac{(-1)^{i}}{\alpha_{i}} \frac{\partial \Psi_{\varepsilon, 1}}{\partial x_{i}}=\left\langle\frac{\partial \Psi_{\varepsilon, 1}}{\partial v}, \frac{\partial P \delta_{i}}{\partial x_{i}}\right\rangle .
$$

Using Proposition 3.2, some computations yield

$$
\frac{\partial \Psi_{\varepsilon, 1}}{\partial \alpha_{i}}=-2 S_{4} \beta_{i}+V_{\alpha_{i}}(\varepsilon, \alpha, \lambda, x),
$$

with $\beta=\left(\beta_{1}, \beta_{2}\right)$ where $\beta_{i}=\alpha_{i}-1 / K\left(y_{i}\right)^{\frac{1}{2}}$ and $V_{\alpha_{i}}$ is a smooth function which satisfies

$$
V_{\alpha_{i}}=O\left(\beta_{i}^{2}+\varepsilon \log \lambda_{i}+\frac{1}{\lambda_{i}^{2}}+\left|x_{i}-y_{i}\right|^{2}\right) .
$$

In the same way, using Proposition 3.3, we get

$$
\begin{aligned}
\frac{\partial \Psi_{\varepsilon, 1}}{\partial \lambda_{i}}= & \frac{1}{K\left(y_{i}\right)}\left(\frac{\varepsilon K\left(x_{i}\right) S_{4}}{4 \lambda_{i}}+\frac{c_{2}}{4}\left(\frac{\Delta K\left(x_{i}\right)}{3 K\left(x_{i}\right)}+4 H\left(x_{i}, x_{i}\right)\right) \frac{1}{\lambda_{i}^{3}}\right) \\
& +\frac{c_{2}}{2\left(K\left(y_{1}\right) K\left(y_{2}\right)\right)^{1 / 2}} \frac{1}{\lambda_{i}} \frac{G\left(x_{1}, x_{2}\right)}{\lambda_{1} \lambda_{2}}+V_{\lambda_{i}}(\varepsilon, \alpha, \lambda, x),
\end{aligned}
$$


where $c_{2}$ and $c_{3}$ are defined in Proposition 3.3 and $V_{\lambda_{i}}$ is a smooth function satisfying

$$
V_{\lambda_{i}}=O\left[\frac{1}{\lambda_{i}}\left(\frac{1}{\lambda_{i}^{3}}+\frac{\left|x_{i}-y_{i}\right|^{2}}{\lambda_{i}^{2}}+\varepsilon^{2} \log \lambda_{i}+\frac{\varepsilon \log \lambda_{i}}{\lambda_{i}^{2}}\right)+\left(|\beta|+\left|x_{i}-y_{i}\right|^{2}\right)\left(\frac{\varepsilon}{\lambda_{i}}+\frac{1}{\lambda_{i}^{3}}\right)\right] \text {. }
$$

Lastly, using Proposition 3.4, we have

$$
\frac{\partial \Psi_{\varepsilon, 1}}{\partial x_{i}}=-c_{3} \nabla K\left(x_{i}\right)+V_{x_{i}}(\varepsilon, \alpha, \lambda, x),
$$

where $V_{x_{i}}$ is a smooth function such that

$$
V_{x_{i}}=O\left(\frac{1}{\lambda_{i}}+\left(|\beta|+\varepsilon \log \lambda_{i}+\left|x_{i}-y_{i}\right|^{2}\right)\left|x_{i}-y_{i}\right|\right) .
$$

Notice that these estimates imply

$$
\frac{\partial \Psi_{\varepsilon, 1}}{\partial \alpha_{i}}=O\left(|\beta|+\varepsilon \log \lambda_{i}+\frac{1}{\lambda_{i}^{2}}+\left|x_{i}-y_{i}\right|^{2}\right), \frac{\partial \Psi_{\varepsilon, 1}}{\partial \lambda_{i}}=O\left(\frac{1}{\lambda_{i}^{3}}+\frac{\varepsilon}{\lambda_{i}}\right), \frac{\partial \Psi_{\varepsilon, 1}}{\partial x_{i}}=O\left(\left|x_{i}-y_{i}\right|+\frac{1}{\lambda_{i}}\right) .
$$

The solution of the system in $A, B$ and $C$ shows that

$A_{i}=O\left(|\beta|+\varepsilon \log \lambda_{i}+\frac{1}{\lambda_{i}^{2}}+\left|x_{i}-y_{i}\right|^{2}\right), \quad B_{i}=O\left(\frac{1}{\lambda_{i}}+\varepsilon \lambda_{i}\right), \quad C_{i}=O\left(\frac{\left|x_{i}-y_{i}\right|}{\lambda_{i}^{2}}+\frac{1}{\lambda_{i}^{3}}\right)$.

This allows us to evaluate the right hand side in the equations $\left(E_{\lambda_{i}}\right)$ and $\left(E_{x_{i}}\right)$, namely

$$
\begin{aligned}
& B_{i}\left\langle\frac{\partial^{2} P \delta_{i}}{\partial \lambda_{i}^{2}}, \bar{v}\right\rangle+\sum_{j=1}^{4} C_{i j}\left\langle\frac{\partial^{2} P \delta_{i}}{\partial x_{i}^{j} \partial \lambda_{i}}, \bar{v}\right\rangle=O\left(\left(\frac{1}{\lambda_{i}^{3}}+\frac{\varepsilon}{\lambda_{i}}+\frac{\left|y_{i}-x_{i}\right|}{\lambda_{i}^{2}}\right)\|\bar{v}\|\right), \\
& B_{i}\left\langle\frac{\partial^{2} P \delta_{i}}{\partial \lambda_{i} \partial x_{i}}, \bar{v}\right\rangle+\sum_{j=1}^{4} C_{i j}\left\langle\frac{\partial^{2} P \delta_{i}}{\partial x_{i}^{j} \partial x_{i}}, \bar{v}\right\rangle=O\left(\left(\frac{1}{\lambda_{i}}+\varepsilon \lambda_{i}+\left|x_{i}-y_{i}\right|\right)\|\bar{v}\|\right),
\end{aligned}
$$

where we have used the following estimates

$$
\left\|\frac{\partial^{2} P \delta_{i}}{\partial \lambda_{i}^{2}}\right\|=O\left(\frac{1}{\lambda_{i}^{2}}\right) ; \quad\left\|\frac{\partial^{2} P \delta_{i}}{\partial x_{i} \partial \lambda_{i}}\right\|=O(1) ; \quad\left\|\frac{\partial^{2} P \delta_{i}}{\partial x_{i}^{2}}\right\|=O\left(\lambda_{i}^{2}\right) .
$$

Now, we consider a point $\left(y_{1}, y_{2}\right) \in S_{+}^{4} \times S_{+}^{4}$ such that $y_{1}$ and $y_{2}$ are nondegenerate critical points of $K$. We set

$$
\frac{1}{\lambda_{i}}=\varepsilon^{\frac{1}{2}} \Lambda_{i}\left(1+\zeta_{i}\right) ; \quad x_{i}=y_{i}+\xi_{i},
$$

where $\zeta_{i} \in \mathbb{R}, \xi_{i} \in \mathbb{R}^{4}$ are assumed to be small and for $i, j \in 1,2, \Lambda_{i}=\Lambda_{i}\left(y_{i}\right)$ verifies

$$
c_{2} \Lambda_{i}^{2}\left(\frac{\Delta K\left(y_{i}\right)}{3 K\left(y_{i}\right)}+4 H\left(y_{i}, y_{i}\right)\right)+S_{4} K\left(y_{i}\right)+c_{2} \Lambda_{i} \Lambda_{j}\left(\frac{K\left(y_{i}\right)}{K\left(y_{j}\right)}\right)^{1 / 2} G\left(y_{i}, y_{j}\right)=0 .
$$

With these changes of variables and using $(4.11),\left(E_{\alpha_{i}}\right)$ is equivalent to

$$
\beta_{i}=V_{\alpha_{i}}(\varepsilon, \beta, \zeta, \xi)=O\left(\beta^{2}+\varepsilon|\log \varepsilon|+|\xi|^{2}\right) .
$$

Now, using (4.13), we show by an easy computation

$$
\frac{\varepsilon K\left(y_{i}+\xi_{i}\right) S_{4}}{4 \lambda_{i}}+\frac{c_{2}}{4}\left(\frac{\Delta K\left(y_{i}+\xi_{i}\right)}{3 K\left(y_{i}+\xi_{i}\right)}+4 H\left(y_{i}+\xi_{i}, y_{i}+\xi_{i}\right)\right) \frac{1}{\lambda_{i}^{3}}
$$




$$
\begin{aligned}
& +\frac{c_{2}}{2\left(K\left(y_{j}\right)\right)^{1 / 2}} \frac{1}{\lambda_{i}} \frac{G\left(y_{1}+\xi_{1}, y_{2}+\xi_{2}\right)}{\lambda_{1} \lambda_{2}} \\
= & K\left(y_{i}\right) \frac{\varepsilon^{3 / 2} S_{4}}{4} \Lambda_{i}\left(1+\zeta_{i}\right)+\frac{c_{2}}{4} \varepsilon^{3 / 2} \Lambda_{i}^{3}\left(1+3 \zeta_{i}\right)\left(\frac{\Delta K\left(y_{i}\right)}{3 K\left(y_{i}\right)}+4 H\left(y_{i}, y_{i}\right)\right. \\
& \left.+\left(\frac{1}{3 K\left(y_{i}\right)} \nabla \Delta K\left(y_{i}\right)+8 \frac{\partial H}{\partial x_{i}}\left(y_{i}, y_{i}\right)\right) \xi_{i}\right)+c_{2} \varepsilon^{3 / 2} \Lambda_{i}^{2} \Lambda_{j} \frac{\left(1+2 \zeta_{i}\right)\left(1+\zeta_{j}\right)}{K\left(y_{j}\right)^{1 / 2}} G\left(y_{i}, y_{j}\right) \\
& +\frac{\varepsilon^{3 / 2} c_{2} \Lambda_{i}^{2} \Lambda_{j}}{K\left(y_{j}\right)^{1 / 2}} \frac{\partial G\left(y_{i}, y_{j}\right)}{\partial x_{1}} \xi_{i}+\frac{c_{2} \varepsilon^{3 / 2} \Lambda_{i}^{2} \Lambda_{j}}{K\left(y_{j}\right)^{1 / 2}} \frac{\partial G\left(y_{i}, y_{j}\right)}{\partial x_{2}} \xi_{j}+O\left(\varepsilon^{3 / 2}\left(\zeta_{i}^{2}+\left|\xi_{i}\right|^{2}\right)\right) \\
= & \varepsilon^{3 / 2}\left[\frac{\Lambda_{i}^{3} c_{2}}{2}\left(\frac{\Delta K\left(y_{i}\right)}{3 K\left(y_{i}\right)}+4 H\left(y_{i}, y_{i}\right)\right)+\frac{c_{2} \Lambda_{i}^{2} \Lambda_{j}}{K\left(y_{j}\right)^{1 / 2}} G\left(y_{i}, y_{j}\right)\right] \zeta_{i}+\frac{\varepsilon^{3 / 2} c_{2} \Lambda_{i}^{2} \Lambda_{j}}{K\left(y_{j}\right)^{1 / 2}} G\left(y_{i}, y_{j}\right) \zeta_{j} \\
& +\varepsilon^{3 / 2}\left[\frac{\Lambda_{i}^{3} c_{2}}{4}\left(\frac{1}{3 K\left(y_{i}\right)} \nabla(\Delta K)\left(y_{i}\right)+8 \frac{\partial H}{\partial x_{i}}\left(y_{i}, y_{i}\right)\right)+\frac{c_{2} \Lambda_{i}^{2} \Lambda_{j}}{K\left(y_{j}\right)^{1 / 2}} \frac{\partial G\left(y_{i}, y_{j}\right)}{\partial x_{1}}\right] \xi_{i} \\
& +\frac{c_{2} \varepsilon^{3 / 2} \Lambda_{i}^{2} \Lambda_{j}}{K\left(y_{j}\right)^{1 / 2}} \frac{\partial G\left(y_{i}, y_{j}\right)}{\partial x_{2}} \xi_{j}+O\left(\varepsilon^{3 / 2}\left(\zeta_{i}^{2}+\left|\xi_{i}\right|^{2}\right)\right) .
\end{aligned}
$$

This implies that $\left(E_{\lambda_{i}}\right)$ is equivalent, on account of (4.14) and (5.9), to

$$
\begin{aligned}
& {\left[\frac{\Lambda_{i}^{3} c_{2}}{2}\left(\frac{\Delta K\left(y_{i}\right)}{3 K\left(y_{i}\right)}+4 H\left(y_{i}, y_{i}\right)\right)+\frac{c_{2} \Lambda_{i}^{2} \Lambda_{j}}{K\left(y_{j}\right)^{1 / 2}} G\left(y_{i}, y_{j}\right)\right] \zeta_{i}+\frac{c_{2} \Lambda_{i}^{2} \Lambda_{j}}{K\left(y_{j}\right)^{1 / 2}} G\left(y_{i}, y_{j}\right) \zeta_{j}} \\
& +\left[\frac{\Lambda_{i}^{3} c_{2}}{4}\left(\frac{1}{3 K\left(y_{i}\right)} \nabla(\Delta K)\left(y_{i}\right)+8 \frac{\partial H}{\partial x_{i}}\left(y_{i}, y_{i}\right)\right)+\frac{c_{2} \Lambda_{i}^{2} \Lambda_{j}}{K\left(y_{j}\right)^{1 / 2}} \frac{\partial G\left(y_{i}, y_{j}\right)}{\partial x_{1}}\right] \xi_{i} \\
& +\frac{c_{2} \Lambda_{i}^{2} \Lambda_{j}}{K\left(y_{j}\right)^{1 / 2}} \frac{\partial G\left(y_{i}, y_{j}\right)}{\partial x_{2}} \xi_{j}=V_{\lambda_{i}}(\varepsilon, \beta, \zeta, \xi)=O\left(|\beta|^{2}+\zeta_{2}^{2}+|\xi|^{2}+\varepsilon^{1 / 2}\right) .
\end{aligned}
$$

Lastly, using (4.15), (4.16) and (5.11), we see that $\left(E_{x_{i}}\right)$ is equivalent to

$$
D^{2} K\left(y_{i}\right) \xi_{i}=V_{x_{i}}(\varepsilon, \beta, \zeta, \xi)=O\left(\varepsilon^{1 / 2}+|\beta|^{2}+|\zeta|^{2}+|\xi|^{2}\right) .
$$

We remark that $V_{\alpha_{i}}, V_{\lambda_{i}}$ and $V_{x_{i}}$ are smooth functions. This system may be written as

$$
\left\{\begin{array}{l}
\beta=V(\varepsilon, \beta, \zeta, \xi), \\
L(\zeta, \xi)=W(\varepsilon, \beta, \zeta, \xi),
\end{array}\right.
$$

where $L$ is a fixed linear operator on $\mathbb{R}^{10}$ defined by (5.15) and (4.21) and $V, W$ are smooth functions satisfying

$$
\left\{\begin{array}{l}
V(\varepsilon, \beta, \zeta, \xi)=O\left(\varepsilon^{1 / 2}+|\beta|^{2}+|\xi|^{2}\right) \\
W(\varepsilon, \beta, \zeta, \xi)=O\left(\varepsilon^{1 / 2}+|\beta|^{2}+|\zeta|^{2}+|\xi|^{2}\right) .
\end{array}\right.
$$

Moreover, a simple computation shows that the determinant of $L$ is not equal to zero. Hence $L$ is invertible, and Brouwer's fixed point theorem shows that (4.22) has a solution $\left(\beta^{\varepsilon}, \zeta^{\varepsilon}, \xi^{\varepsilon}\right)$ for $\varepsilon$ small enough, such that

$$
\left|\beta^{\varepsilon}\right|=O\left(\varepsilon^{1 / 2}\right) ; \quad\left|\zeta^{\varepsilon}\right|=O\left(\varepsilon^{1 / 2}\right) ; \quad\left|\xi^{\varepsilon}\right|=O\left(\varepsilon^{1 / 2}\right) .
$$


Hence, we have constructed $m^{\varepsilon}=\left(\alpha_{1}^{\varepsilon}, \alpha_{2}^{\varepsilon}, \lambda_{1}^{\varepsilon}, \lambda_{2}^{\varepsilon}, x_{1}^{\varepsilon}, x_{2}^{\varepsilon}\right)$ such that $u_{\varepsilon}:=\alpha_{1}^{\varepsilon} P \delta_{\left(x_{1}^{\varepsilon}, \lambda_{1}^{\varepsilon}\right)}-$ $\alpha_{2}^{\varepsilon} P \delta_{\left(x_{2}^{\varepsilon}, \lambda_{2}^{\varepsilon}\right)}+\overline{v_{\varepsilon}}$, satisfies (4.2)-(4.6). Therefore, by Proposition 4.1, $u_{\varepsilon}$ is a critical point of $I_{\varepsilon}$, i.e., $u_{\varepsilon}$ satisfies

$$
-\Delta u_{\varepsilon}+2 u_{\varepsilon}=K\left|u_{\varepsilon}\right|^{2-\varepsilon} u_{\varepsilon} \quad \text { in } S_{+}^{4}, \quad \partial u_{\varepsilon} / \partial v=0 \text { on } \partial S_{+}^{4} .
$$

Hence, the proof of Theorem 1.1 is thereby completed.

5. Proof of Theorem 1.2. As in the proof of Theorem 1.1, we introduce the set

$$
\begin{aligned}
M_{\varepsilon, 2}= & \left\{m=\left(\alpha, \lambda, x_{1}, x_{2}, v\right) \in \mathbb{R}^{2} \times\left(\mathbb{R}_{+}^{*}\right)^{2} \times S_{+}^{4} \times S_{+}^{4} \times H^{1}\left(S_{+}^{4}\right):\left|\frac{\alpha_{i}^{2} K\left(x_{i}\right)}{\alpha_{j}^{2} K\left(x_{j}\right)}-1\right|<v_{0},\right. \\
& \left.\lambda_{i}>\frac{1}{v_{0}}, \varepsilon \log \lambda_{i}<v_{0}, d_{0}<\frac{\lambda_{1}}{\lambda_{2}^{3}}<\frac{1}{d_{0}}, \lambda_{1}\left|x_{1}-x_{2}\right|<d_{0}^{\prime}, v \in E_{(x, \lambda)},\|v\|<v_{0}\right\},
\end{aligned}
$$

where $d_{0}$ and $d_{0}^{\prime}$ are suitable positive constants, $v_{0}$ is a small positive constant. Let us define the functional

$$
\Psi_{\varepsilon, 2}: M_{\varepsilon, 2} \rightarrow \mathbb{R} ; \quad m=(\alpha, \lambda, x, v) \mapsto I_{\varepsilon}\left(\alpha_{1} P \delta_{\left(x_{1}, \lambda_{1}\right)}-\alpha_{2} P \delta_{\left(x_{2}, \lambda_{2}\right)}+v\right) .
$$

Let $m=(\alpha, \lambda, x, v) \in M_{\varepsilon, 2} . m$ is a critical point of $\Psi_{\varepsilon, 2}$ if and only if $u=\alpha_{1} P \delta_{1}-\alpha_{2} P \delta_{2}+v$ is a critical point of $I_{\varepsilon}$, i.e. if and only if there exists $(A, B, C) \in \mathbb{R}^{2} \times \mathbb{R}^{2} \times\left(\mathbb{R}^{4}\right)^{2}$ such that the system (4.2)-(4.5) holds. Once $\bar{v}$ is defined by Proposition 4.2, we estimate the corresponding numbers $A, B$ and $C$ by taking the scalar product in $H^{1}\left(S_{+}^{4}\right)$ of $\left(E_{v}\right)$ with $P \delta_{i}, \partial P \delta_{i} / \partial \lambda_{i}$ and $\partial P \delta_{i} / \partial x_{i}$ respectively. Thus we get a quasi-diagonal system whose coefficients are given by (we remark that in this region $\varepsilon_{12}$ is of the order of $\lambda_{2}^{-2}$ )

$$
\begin{aligned}
& \int_{\mathbb{R}_{+}^{4}} \nabla P \delta_{i} \nabla P \delta_{j}=S_{4} \delta_{i j}+O\left(\frac{1}{\lambda_{2}^{2}}\right) ; \quad \int_{\mathbb{R}_{+}^{4}} \nabla P \delta_{i} \nabla \frac{\partial P \delta_{j}}{\partial \lambda_{j}}=O\left(\text { if }(i=j) \frac{1}{\lambda_{i}^{3}} ; \text { if }(i \neq j) \frac{1}{\lambda_{j} \lambda_{2}^{2}}\right), \\
& \int_{\mathbb{R}_{+}^{4}} \nabla P \delta_{i} \nabla \frac{\partial P \delta_{j}}{\partial x_{j}}=O\left(\text { if }(i=j) \frac{1}{\lambda_{i}^{2}} ; \text { if }(i \neq j) \frac{1}{\lambda_{1}}\right) ;\left.\quad \int_{\mathbb{R}_{+}^{4}} \nabla \frac{\partial P \delta_{i}}{\partial \lambda_{i}}\right|^{2}=\frac{\Gamma_{1}}{\lambda_{i}^{2}}+O\left(\frac{1}{\lambda_{i}^{3}}\right), \\
& \int_{\mathbb{R}_{+}^{4}} \nabla \frac{\partial P \delta_{1}}{\partial \lambda_{1}} \nabla \frac{\partial P \delta_{2}}{\partial \lambda_{2}}=O\left(\frac{1}{\lambda_{1} \lambda_{2}^{3}}\right), \int_{\mathbb{R}_{+}^{4}} \nabla \frac{\partial P \delta_{1}}{\partial \lambda_{1}} \nabla \frac{\partial P \delta_{2}}{\partial x_{2}}=O\left(\frac{1}{\lambda_{1}^{2}}\right), \int_{\mathbb{R}_{+}^{4}} \nabla \frac{\partial P \delta_{2}}{\partial \lambda_{2}} \nabla \frac{\partial P \delta_{1}}{\partial x_{1}}=O\left(\frac{1}{\lambda_{2}^{4}}\right), \\
& \int_{\mathbb{R}_{+}^{4}} \nabla \frac{\partial P \delta_{i}}{\partial \lambda_{i}} \nabla \frac{\partial P \delta_{i}}{\partial x_{i}}=O\left(\frac{1}{\lambda_{i}^{3}}\right), \int_{\mathbb{R}_{+}^{4}} \nabla \frac{\partial P \delta_{i}}{\partial x_{i}} \nabla \frac{\partial P \delta_{j}}{\partial x_{j}}=\Gamma_{2} \lambda_{i}^{2} \delta_{i j}+O\left(\text { if }(i=j) \frac{1}{\lambda_{i}} ; \text { if }(i \neq j) 1\right) .
\end{aligned}
$$

On the other hand side, $\Psi_{\varepsilon, 2}$ satisfies (4.10). By Proposition 3.2, (4.11) and (4.12) are satisfied with $\lambda_{2}$ instead of $\lambda$. In the same way we get

$$
\begin{aligned}
& \frac{\partial \Psi_{\varepsilon, 2}}{\partial \lambda_{1}}=\frac{1}{K(\bar{y})}\left(\frac{S_{4}}{4} \frac{\varepsilon}{\lambda_{1}}-c_{2} \frac{\lambda_{2}}{\lambda_{1}^{2}}\right)+V_{\lambda_{1}}(\varepsilon, \alpha, \lambda, x), \\
& \frac{\partial \Psi_{\varepsilon, 2}}{\partial \lambda_{2}}=\frac{1}{K(\bar{y})}\left(\frac{S_{4}}{4} \frac{\varepsilon}{\lambda_{2}}+\frac{c_{2}}{4}\left(\frac{\Delta K\left(x_{2}\right)}{3 K\left(x_{2}\right)}+4 H\left(x_{2}, x_{2}\right)\right) \frac{1}{\lambda_{2}^{3}}+\frac{c_{2}}{\lambda_{1}}\right)+V_{\lambda_{2}}(\varepsilon, \alpha, \lambda, x),
\end{aligned}
$$


where $V_{\lambda_{i}}$ is a smooth function verifying

(5.4)

$$
V_{\lambda_{i}}=O\left\{\frac{1}{\lambda_{i}}\left(\varepsilon^{2} \log \lambda_{i}+\frac{\varepsilon \log \lambda_{i}}{\lambda_{i}^{2}}+\left(|\beta|+\varepsilon+\left|x_{i}-\bar{y}\right|^{2}\right)\left(\varepsilon+\frac{1}{\lambda_{2}^{2}}\right)+\sum_{q=1,2} \frac{\left|x_{q}-\bar{y}\right|^{2}}{\lambda_{q}^{2}}+\frac{1}{\lambda_{2}^{3}}\right)\right\} .
$$

Lastly, we have

$$
\frac{\partial \Psi_{\varepsilon, 2}}{\partial x_{i}}=-\frac{c_{3}}{K(\bar{y})^{2}} \nabla K\left(x_{i}\right)+V_{x_{i}}(\varepsilon, \alpha, \lambda, x),
$$

where $V_{x_{i}}$ is a smooth function verifying

$$
V_{x_{i}}=O\left(\varepsilon^{2} \lambda_{i} \log \lambda_{i}+\frac{1}{\lambda_{i}}+\lambda_{i} \sum_{q=1,2} \frac{\left|x_{q}-\bar{y}\right|^{2}}{\lambda_{q}^{2}}+\left(|\beta|+\varepsilon+\left|x_{i}-\bar{y}\right|^{2}\right)\left|x_{i}-\bar{y}\right|\right) .
$$

Notice that these estimates imply

$$
\begin{aligned}
& \frac{\partial \Psi_{\varepsilon, 2}}{\partial \alpha_{i}}=O\left(|\beta|+\frac{1}{\lambda_{2}^{2}}+\varepsilon \log \lambda_{2}+\left|x_{i}-\bar{y}\right|^{2}\right) ; \quad \frac{\partial \Psi_{\varepsilon, 2}}{\partial \lambda_{i}}=O\left(\frac{1}{\lambda_{i} \lambda_{2}^{2}}+\frac{\varepsilon}{\lambda_{i}}\right), \\
& \frac{\partial \Psi_{\varepsilon, 2}}{\partial x_{i}}=O\left(\frac{1}{\lambda_{i}}+\varepsilon^{2} \lambda_{i} \log \lambda_{i}+\left|x_{i}-\bar{y}\right|+\lambda_{i} \sum_{q=1,2} \frac{\left|x_{q}-\bar{y}\right|^{2}}{\lambda_{q}^{2}}\right) .
\end{aligned}
$$

The solution of the system in $A, B$ and $C$ shows that

$$
\left\{\begin{array}{l}
A_{i}=O\left(|\beta|+\frac{1}{\lambda_{2}^{2}}+\varepsilon \log \lambda_{2}+\left|x_{i}-\bar{y}\right|^{2}\right), \quad B_{i}=O\left(\frac{\lambda_{i}}{\lambda_{2}^{2}}+\varepsilon \lambda_{i}\right), \\
C_{1}=O\left(\frac{1}{\lambda_{1}^{3}}+\frac{\varepsilon^{2} \log \lambda_{1}}{\lambda_{1}}+\frac{\left|x_{1}-\bar{y}\right|}{\lambda_{1}^{2}}+\frac{\left|x_{2}-\bar{y}\right|^{2}}{\lambda_{1} \lambda_{2}^{2}}\right) ; \quad C_{2}=O\left(\frac{1}{\lambda_{2}^{3}}+\frac{\varepsilon^{2} \log \lambda_{2}}{\lambda_{2}}+\frac{\left|x_{2}-\bar{y}\right|}{\lambda_{2}^{2}}\right) .
\end{array}\right.
$$

This makes us able to evaluate the right hand side in the equations $\left(E_{\lambda_{i}}\right)$ and $\left(E_{x_{i}}\right)$, namely as in the proof of Theorem 1.1, we get

$$
\begin{aligned}
& B_{1}\left\langle\frac{\partial^{2} P \delta_{1}}{\partial \lambda_{1}^{2}}, \bar{v}\right\rangle+\sum_{j=1}^{4} C_{1 j}\left\langle\frac{\partial^{2} P \delta_{1}}{\partial x_{1}^{j} \partial \lambda_{1}}, \bar{v}\right\rangle=O\left(\left(\frac{\varepsilon}{\lambda_{1}}+\frac{\left|x_{1}-\bar{y}\right|}{\lambda_{1}^{2}}+\frac{1}{\lambda_{1} \lambda_{2}^{2}}\right)\|\bar{v}\|\right) \\
& B_{2}\left\langle\frac{\partial^{2} P \delta_{2}}{\partial \lambda_{1}^{2}}, \bar{v}\right\rangle+\sum_{j=1}^{4} C_{2 j}\left\langle\frac{\partial^{2} P \delta_{2}}{\partial x_{2}^{j} \partial \lambda_{2}}, \bar{v}\right\rangle=O\left(\left(\frac{1}{\lambda_{2}^{2}}+\frac{\varepsilon}{\lambda_{2}}+\frac{\left|\bar{y}-x_{2}\right|}{\lambda_{2}^{2}}\right)\|\bar{v}\|\right) \\
& B_{1}\left\langle\frac{\partial^{2} P \delta_{1}}{\partial \lambda_{1} \partial x_{1}}, \bar{v}\right\rangle+\sum_{j=1}^{4} C_{1 j}\left\langle\frac{\partial^{2} P \delta_{1}}{\partial x_{1}^{j} \partial x_{1}}, \bar{v}\right\rangle=O\left(\lambda_{2}\|\bar{v}\|\right) \\
& B_{2}\left\langle\frac{\partial^{2} P \delta_{2}}{\partial \lambda_{2} \partial x_{2}}, \bar{v}\right\rangle+\sum_{j=1}^{4} C_{2 j}\left\langle\frac{\partial^{2} P \delta_{2}}{\partial x_{2}^{j} \partial x_{2}}, \bar{v}\right\rangle=O(\|\bar{v}\|)
\end{aligned}
$$

Now, we consider a point $\bar{y}$ in $S_{+}^{4}$ such that $\bar{y}$ is a nondegenerate critical point of $K$. We set

$$
\frac{1}{\lambda_{2}}=\left(\frac{S_{4}}{4 c_{2}}\right)^{1 / 2} \Lambda(\bar{y})\left(1+\zeta_{2}\right) \varepsilon^{1 / 2} ; \quad \frac{\lambda_{2}^{3}}{\lambda_{1}}=\frac{1}{\Lambda(\bar{y})^{2}}\left(1+\zeta_{1}\right), \quad x_{i}=\bar{y}+\xi_{i},
$$

where $\zeta_{i} \in \mathbb{R}$ and $\xi_{i} \in \mathbb{R}^{4}$ are assumed to be small and 


$$
\Lambda(\bar{x}):=\bar{\Lambda}=\sqrt{2}\left(-H(\bar{y}, \bar{y})-\frac{\Delta K(\bar{y})}{12 K(\bar{y})}\right)^{-1 / 2} .
$$

With these changes of variables, $\left(E_{\alpha_{i}}\right)$ is equivalent to (4.19). Now, using (5.2), we show by an easy computation that

$$
\begin{aligned}
\frac{S_{4}}{4} \varepsilon-c_{2} \frac{\lambda_{2}}{\lambda_{1}} & =\frac{S_{4}}{4} \varepsilon-\frac{c_{2}}{\bar{\Lambda}^{2}} \frac{1}{\lambda_{2}^{2}}\left(1+\zeta_{1}\right)=\frac{S_{4}}{4} \varepsilon-\frac{S_{4}}{4}\left(1+\zeta_{1}\right)\left(1+\zeta_{2}\right)^{2} \varepsilon \\
& =-\frac{S_{4}}{4}\left(\zeta_{1}+2 \zeta_{2}\right) \varepsilon+O\left(\varepsilon\left(\zeta_{1}^{2}+\zeta_{2}^{2}\right)\right) .
\end{aligned}
$$

Thus, $\left(E_{\lambda_{1}}\right)$ is equivalent, on account of (5.4) and (5.8), to

$$
\zeta_{1}+2 \zeta_{2}=V_{\lambda_{1}}(\varepsilon, \beta, \zeta, \xi)=O\left(\varepsilon|\log \varepsilon|+|\beta|+|\zeta|^{2}+|\xi|^{2}\right)
$$

Using (5.3), we have

$$
\begin{aligned}
& \frac{S_{4}}{4} \varepsilon+\frac{c_{2}}{4}\left(4 H\left(\bar{y}+\xi_{2}, \bar{y}+\xi_{2}\right)+\frac{\Delta K\left(\bar{y}+\xi_{2}\right)}{3 K\left(\bar{y}+\xi_{2}\right)}\right) \frac{1}{\lambda_{2}^{2}}+c_{2} \frac{\lambda_{2}}{\lambda_{1}} \\
& =\frac{S_{4}}{4} \varepsilon+\frac{S_{4}}{4} \bar{\Lambda}^{2}\left(1+\zeta_{2}\right)^{2} \varepsilon\left(H(\bar{y}, \bar{y})+\frac{\Delta K(\bar{y})}{12 K(\bar{y})}+2 \frac{\partial H}{\partial a}(\bar{y}, \bar{y}) \xi_{2}+\frac{\nabla \Delta K(\bar{y}) \xi_{2}}{12 K(\bar{y})}+O\left(\left|\xi_{2}\right|^{2}\right)\right) \\
& \quad+\frac{\varepsilon S_{4}}{4}\left(1+\zeta_{1}\right)\left(1+\zeta_{2}\right)^{2} \\
& =\frac{S_{4}}{4} \varepsilon\left(\zeta_{1}-2 \zeta_{2}+\frac{\bar{\Lambda}^{2}}{12 K(\bar{y})} \nabla \Delta K(\bar{y}) \xi_{2}+2 \bar{\Lambda}^{2} \frac{\partial H}{\partial a}(\bar{y}, \bar{y}) \xi_{2}\right)+O\left(\varepsilon\left(|\zeta|^{2}+\left|\xi_{2}\right|^{2}\right)\right) .
\end{aligned}
$$

This implies that $\left(E_{\lambda_{2}}\right)$ is equivalent, on account of (5.4) and (5.8), to

$$
\zeta_{1}-2 \zeta_{2}+\bar{\Lambda}^{2}\left(\frac{1}{12 K(\bar{y})} \nabla \Delta K(\bar{y})+2 \frac{\partial H}{\partial a}(\bar{y}, \bar{y})\right) \xi_{2}=V_{\lambda_{2}}(\varepsilon, \beta, \zeta, \xi),
$$

where $V_{\lambda_{2}}(\varepsilon, \beta, \zeta, \xi)=O\left(\varepsilon^{1 / 2}+|\beta|+|\zeta|^{2}+|\xi|^{2}\right)$. Using (5.5), (5.6) and (5.10), we see that $\left(E_{x_{i}}\right)$ is equivalent to

$$
D^{2} K(\bar{y}) \xi_{i}=V_{x_{i}}(\varepsilon, \beta, \zeta, \xi)=O\left((\varepsilon|\ln \varepsilon|)^{1 / 2}+|\beta|^{2}+|\xi|^{2}\right)
$$

We remark that $V_{\alpha_{i}}, V_{\lambda_{i}}$ and $V_{x_{i}}$ are smooth functions. This system may be written as

$$
\left\{\begin{array}{l}
\beta=V(\varepsilon, \beta, \zeta, \xi) \\
L_{2}(\zeta, \xi)=W_{2}(\varepsilon, \beta, \zeta, \xi)
\end{array}\right.
$$

where $L_{2}$ is a fixed linear operator of $\mathbb{R}^{10}$ defined by

$$
L_{2}(\zeta, \xi)=\left(\zeta_{1}+2 \zeta_{2} ; \zeta_{1}-2 \zeta_{2}+\bar{\Lambda}^{2}\left(\frac{1}{12 K(\bar{y})} \nabla \Delta K(\bar{y})+\frac{\partial H(\bar{y}, \bar{y})}{\partial a}\right) \xi_{2} ; D^{2} K(\bar{y}) \xi_{1} ; D^{2} K(\bar{y}) \xi_{2}\right),
$$

and $V, W_{2}$ are smooth functions satisfying

$$
\left\{\begin{array}{l}
V(\varepsilon, \beta, \zeta, \xi)=O\left(\varepsilon|\log \varepsilon|+|\beta|^{2}+|\xi|^{2}\right) \\
W_{2}(\varepsilon, \beta, \zeta, \xi)=O\left(\varepsilon^{1 / 2}+|\beta|^{2}+|\zeta|^{2}+|\xi|^{2}\right) .
\end{array}\right.
$$


$\bar{y}$ is a nondegenerate critical point of $K$ by assumption, $L_{2}$ is invertible, and Brouwer's fixed point theorem shows that (5.15) has a solution $\left(\beta^{\varepsilon}, \zeta^{\varepsilon}, \xi^{\varepsilon}\right)$ for $\varepsilon$ small enough, such that

$$
\left|\beta^{\varepsilon}\right|=O(\varepsilon|\log \varepsilon|), \quad\left|\zeta^{\varepsilon}\right|=O\left(\varepsilon^{1 / 2}\right), \quad\left|\xi^{\varepsilon}\right|=O\left((\varepsilon|\ln \varepsilon|)^{1 / 2}\right) .
$$

By construction, the corresponding $u_{\varepsilon} \in H^{1}\left(S_{+}^{4}\right)$ is a critical point of $I_{\varepsilon}$, i.e. $u_{\varepsilon}$ satisfies $\left(S_{\varepsilon}\right)$. The proof of Theorem 1.2 is thereby completed.

Acknowledgment. The second author gratefully acknowledges the Deanship of Scientific Research at Taibah University on material and moral support in the financing of this research project.

\section{REFERENCES}

[1] A. BAHRI, An invarient for Yamabe-type flows with applications to scalar curvature problems in high dimension, A celebration of J. F. Nash Jr., Duke Math. J. 81 (1996), 323-466.

[2] A. BAHRI, Y. Y. Li AND O. REY, On a variational problem with lack of compactness: The topological effect of the critical points at infinity, Calc. Var. Partial Differential Equations 3 (1995), 67-94.

[ 3 ] M. Ben Ayed, K. El Mehdi And M. Ould Ahmedou, The scalar curvature problem on the four dimensional half sphere, Calc. Var. 22 (2005), 465-482.

[4] M. Ben Ayed, R. GHoudi And K. Ould Bouh, Existence of conformal metrics with prescribed scalar curvature on the four dimensional half sphere, NoDEA Nonlinear Differential Equations Appl. 19 (2012), 629-662.

[ 5 ] G. Bianchi And X. B. PAn, Yamabe equations on half-spaces, Nonlinear Anal. 37 (1999), 161-186.

[6] Z. DJadli, A. Malchiodi AND M. Ould Ahmedou, Prescribing the scalar and the boundary mean curvature on the three dimensional half sphere, J. Geom. Anal. 13 (2003), 233-267.

[ 7 ] R. GHOUd, Blowing up of sign-changing solutions to an elliptic subcritical equation, J. Partial Differ. Equ. 25 (2012), no. 4, 368-388.

[ 8 ] Y. Y. LI, Prescribing scalar curvature on $S^{n}$ and related topics, Part I, J. Differental Equations 120 (1995), 319-410; Part II, existence and compactness, Comm. Pure Appl. Math. 49 (1996), 437-477.

[9] M. Musso And A. Pistoia, Tower of bubbles for almost critical problems in general domains, J. Math. Pures Appl. 93 (2010), no. 1-140.

[10] A. Pistoia And T. Weth, Sign-changing bubble-tower solutions in a slightly subcritical semilinear Dirichlet problem, Ann. Inst. H. Poincaré Anal. Non Linéaire 24 (2007), 325-340.

[11] O. REY, The topological impact of critical points at infinity in a variational problem with lack of compactness: the dimension 3, Adv. Differential Equations 4 (1999), 581-616.

UNIVERSITÉ DE GABÈS

FACULTÉ DES SCIENCES

CITÉ El RIADH, GABÈS

TUNISIA

E-mail address: ghoudi.rabeh@yahoo.fr

\author{
Department of MATHEMATICS \\ COLLEGE OF SCIENCES \\ TAIBAH UNIVERSITY \\ P.O.BOX: 30002 \\ Almadinah Almunawwarah \\ KINGDOM OF SAUdi ARABIA
}

E-mail address: hbouh@taibahu.edu.sa : kamal_bouh@yahoo.fr

http://www.math.tohoku.ac.jp/tmj/Esubmit.html\#article 